\title{
Ferramenta de elaboração de testes de autoavaliação para ambientes de ensino a
}

\section{distância}

\author{
Tool for developing self-assessment tests for distance learning environments
}

Herramienta para desarrollar pruebas de autoevaluación para entornos de aprendizaje a distancia

Recebido: 21/06/2021 | Revisado: 27/06/2021 | Aceito: 01/07/2021 | Publicado: 14/07/2021

\author{
Áquila Jerard Moulin Ditzz \\ ORCID: https://orcid.org/0000-0002-4079-6571 \\ Instituto Federal Fluminense, Brasil \\ E-mail: aquila.ditzz@iff.edu.br \\ Geórgia Regina Rodrigues Gomes \\ ORCID: https://orcid.org/0000-0003-0907-9838 \\ Universidade Federal Fluminense, Brasil \\ E-mail: georgiagomes@id.uff.br
}

\begin{abstract}
Resumo
Atualmente pode ser observado um aumento na utilização de tecnologias da informação e comunicação, bem como o uso da internet. Converge ainda neste ponto a crescente procura por cursos na modalidade de Ensino a Distância (EaD). Neste cenário, existe a demanda por Learning Management Systems (LMS) que forneçam aos usuários, dentre outras características, alternativas para criação e gerenciamento de avaliações do conhecimento. Considerando o que foi disposto, o presente artigo descreve um LMS desenvolvido com o objetivo de permitir a criação de testes compostos de questões objetivas e discursivas, com correção automática e feedback imediato para os alunos, utilizando técnicas de Text Mining. Além disso é apresentado um estudo de caso desenvolvido para aferição dos resultados das correções de avaliações obtidos por intermédio da referida ferramenta, indicando a viabilidade desta e os relatos dos professores e estudantes que utilizaram a ferramenta, bem como uma comparação às avaliações realizadas pelos professores.
\end{abstract}

Palavras-chave: Learning management systems; Ensino a distância; Avaliação; Ensino.

\begin{abstract}
Currently, an increase in the use of information and communication technologies can be observed, as well as the use of the internet. The growing demand for Distance Learning courses also converges at this point. In this scenario, there is a demand for Learning Management Systems (LMS) that provide users, among other features, alternatives for creating and managing knowledge assessments. Considering that, this article describes an LMS, developed with the objective of allowing the creation of tests composed of objective and discursive questions, with automatic correction and immediate feedback to students, using Text Mining techniques. In addition, a case study was applied to measure the results of the corrections of evaluations obtained through this tool, indicating its feasibility and the reports of teachers and students who used the tool, as well the correction performed by the tool compared to assessments carried out by teachers.
\end{abstract}

Keywords: Learning management systems; Distance learning; Assessment; Teaching.

\begin{abstract}
Resumen
Actualmente se observa un incremento en el uso de tecnologías de la información y la comunicación, así como en el uso de internet. La creciente demanda de cursos de Educación a Distancia también converge en este punto. En este escenario, existe una demanda de Learning Management Systems (LMS) que brinden a los usuarios, entre otras características, alternativas para la creación y gestión de evaluaciones de conocimiento. Teniendo en cuenta lo planteado, este artículo describe un LMS desarrollado con el objetivo de permitir la creación de pruebas compuestas por preguntas objetivas y discursivas, con corrección automática y retroalimentación inmediata a los estudiantes, utilizando técnicas de Text Mining. Además, se presenta un caso de estudio desarrollado para medir los resultados de las correcciones de evaluaciones obtenidas a través de esta herramienta, indicando su viabilidad y los informes de los docentes y estudiantes que utilizaron la herramienta, así como una comparación con las evaluaciones realizadas por los docentes.
\end{abstract}

Palabras clave: Learning management systems; Educación a distancia; Evaluación; Enseñando. 


\section{Introdução}

Nas últimas décadas as, devido à ampliação da utilização de Tecnologias da Informação e Comunicação (TIC), principalmente com o uso da internet, a sociedade sofreu grande impacto em várias áreas, como geopolítica, nas relações de trabalho, na comunicação e no ensino aprendizagem, dentre outras (Clementino, 2008; Alves et al., 2016). De forma complementar é possível perceber que com o avanço tecnológico, principalmente com o aumento da utilização de dispositivos móveis e popularização da internet, a sociedade mudou sua forma de se relacionar e realizar suas tarefas cotidianas, inclusive seu modo de aprender (Pinheiro, 2000; Silva \& Gomes, 2016).

Em relatório publicado por CGI.br/NIC.br (2018), em levantamento específico quanto à utilização das TIC no cotidiano, é possível constatar que a maior parte dos lares brasileiros possui algum tipo de TIC, sendo que o acesso à internet está presente em $67 \%$ destes. Neste mesmo levantamento, na pesquisa sobre dispositivos utilizados para realizar acesso à internet é possível constatar que o smartphone é a principal tipo de TIC utilizado para este fim. Neste aspecto a UNESCO (2014), ao definir os objetivos do milênio, por entender que a utilização de smartphones e tablets pode enriquecer e ampliar as oportunidades de aprendizagem, decidiu incluiu a utilização de m-learning no ensino.

Com relação às atividades inerentes ao ensino, a expansão na utilização das TIC e o acesso à internet afetaram diretamente a área da educação, e essas mudanças vão muito além da sala de aula tradicional. Houve também um aumento significativo na oferta de cursos na modalidade denominada Educação a Distância ou Ensino a Distância, conhecida usualmente pela sigla EaD. Em CENSO realizado pela Associação Brasileira de Educação a Distância (ABED) de 2015, é possível observar que o número de estudantes inscritos em cursos à distância foi de 5 milhões, 1,1 milhão a mais de estudantes comparado com o levantamento de 2014, representando um aumento percentual de $28 \%$ no intervalo de um ano.

Com relação aos desafios presentes, tanto no ensino presencial ou a distância, destaca-se a aprendizagem dos alunos. Neste aspecto, Scaico et al. (2012) afirmam que cada indivíduo possui singularidades no processo de aprendizagem, comportando-se de maneira única na forma de absorção e retenção do conhecimento. Porém, quando o estilo de aprendizagem é reconhecido torna-se possível elaborar mecanismos para se obter melhor aproveitamento, tornando o processo de aprendizagem mais eficiente. Neste aspecto Dunlosky et al. (2013) ao analisarem algumas técnicas usualmente utilizadas no processo de aprendizagem, destacam que para a maior parte dos indivíduos, a realização de testes práticos acerca dos temas estudados, apresenta-se como uma das mais eficientes.

Portanto a motivação para o trabalho descrito no presente artigo surge na percepção da necessidade de uma ferramenta de criação e realização de testes práticos, com foco na autoavaliação, permitindo por meio de estratégias de feedback automático que alunos e professores percebam conteúdos que devam ser revisitados em suas práticas de ensino e aprendizagem, sem o caráter punitivo que por vezes as avaliações assumem. Conforme descrito anteriormente, o smartphone é a TIC mais presente nos lares brasileiros, portanto um aspecto que se considerou neste trabalho foi o de que a ferramenta desenvolvida, além de atender à crescente demanda por cursos na modalidade $\mathrm{EaD}$, também estivesse disponível para dispositivos móveis.

\section{Metodologia}

Para a condução da pesquisa, optou-se pelo desenvolvimento de uma nova aplicação que atendesse ao objetivo inicialmente identificado em vez da utilização de uma ferramenta já consolidada no mercado. O desenvolvimento de uma nova aplicação também se justifica por não haver atualmente uma ferramenta que concentre todas as características desejáveis, como produção de questões objetivas e discursivas sem que haja necessidade de interação de terceiros participando na correção destas atividades. 
Uma das principais preocupações acerca do desenvolvimento esteve relacionada com o grande crescimento de dispositivos móveis no Brasil e no mundo. Porém, por preocupações com aspectos de usabilidade e ergonomia, por vezes é mais confortável para o usuário criar e realizar os testes providos pela ferramenta em um laptop ou computador de mesa, por esses motivos optou-se pelo desenvolvimento utilizando design responsivo, que possibilita a adequação automática do conteúdo para diversos dispositivos, como smartphones e computadores pessoais.

\subsection{Ferramenta Desenvolvida}

A ferramenta desenvolvida para este trabalho consiste em um LMS com foco na criação de testes de autoavaliação, adaptado de Foly (2010), intitulado "Uma arquitetura para aferir o conhecimento do aluno em um ambiente de aprendizagem". Para elucidar o leitor acerca do que foi desenvolvido por Foly (2010) e do que foi desenvolvido para o presente trabalho, optou-se pela utilização de um mapa conceitual.

No mapa conceitual representado pela Figura 1, foi utilizado cor azul claro para representar o que havia sido desenvolvido por Foly (2010) e na cor azul escuro as novas funcionalidades implementadas para a ferramenta desenvolvida para este trabalho.

Figura 1 - Mapa Conceitual da ferramenta.

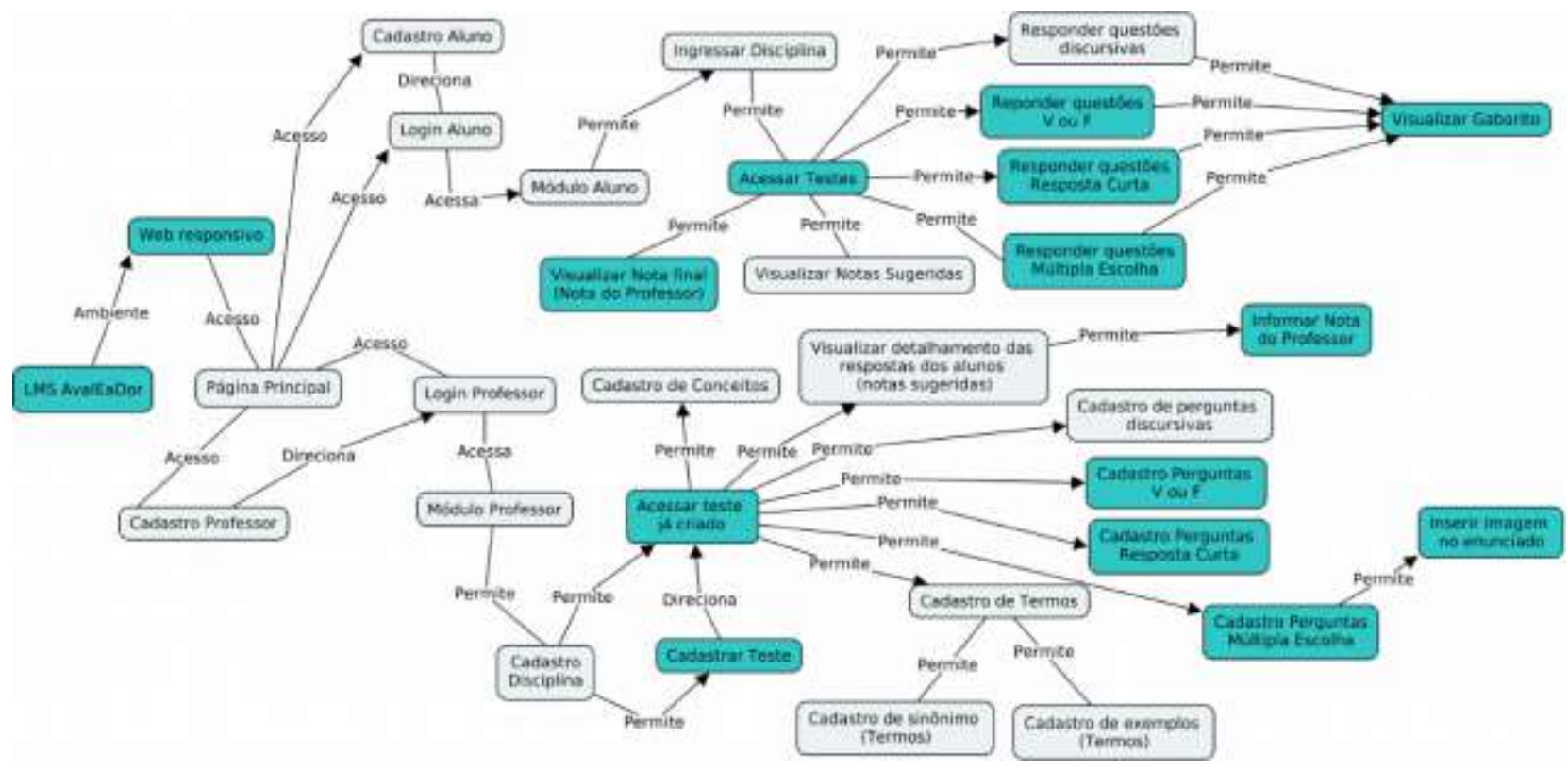

Fonte: Autores.

Fica evidenciado na Figura 1 que no presente trabalho houve a adequação da ferramenta previamente desenvolvida por Foly (2010) para dispositivos móveis, por meio da implementação de responsividade ao projeto, e o acréscimo de novas funcionalidades, permitindo que os professores elaborem atividades mais diversificadas, adequadas aos propósitos por estes especificados.

Para comparação de questões dos tipos "múltipla escolha", "resposta curta" e "verdadeira ou falsa", a resposta informada pelo professor, é comparada com a resposta fornecida pelo aluno. Caso as respostas fornecidas coincidam, o exercício é corrigido como correto e o aluno recebe nota dez $(10,0)$, caso contrário o status da questão é considerado incorreto e o aluno recebe nota zero $(0,0)$. Para os referidos tipos de questão não é possível que o aluno obtenha notas parciais, uma vez que são questões diretas. 
Nas questões do tipo discursiva, a resposta informada pelo aluno é comparada à resposta informada pelo professor, previamente tratada e armazenada na base de conhecimento, para se definir o percentual de acerto do aluno, porém, diferentemente das questões objetivas, esta comparação não é direta, os dados de ambos são tratados, utilizando técnicas de Text Mining e para atribuição de notas é utilizada a equação de Abrangência, definida em Gomes (2006) como:

$$
\text { Abrangência }=\frac{\text { Número de itens certos recuperados de A }}{\text { Número total de itens certos em } B}
$$

Onde o "número de itens certos recuperados de A" equivalem ao número de itens que o sistema recuperou de forma consistente no conjunto de dados do aluno e o "número total de itens certos em B" corresponde ao número de itens certos existentes no texto no conjunto de dados do professor (GOMES, 2006).

As etapas utilizadas no Text Mining necessárias para uma comparação mais flexível, segundo Foly (2010), são:

- Tokenização: Nesta etapa o texto é dividido em pequenos fragmentos, denominados tokens. $\mathrm{O}$ algoritmo utilizado identifica pontos de quebra destes fragmentos, geralmente espaços, pontos e vírgulas, e gera um token por linha;

- $\quad$ Filtragem de Stopwords: Nesta etapa são eliminados os tokens sem relevância, geralmente palavras sem significado semântico, como por exemplo, artigos, preposições e conjunções.

- $\quad$ Análise de Sinonímia: Nesta etapa são buscados sinônimos na base de conhecimento, eliminando eventuais inconsistências, gerando termos únicos para garantir que sinônimos não sejam tratados como termos distintos.

- Stemming: Nesta etapa, são extraídos os radicais dos tokens relevantes o resultado dessa radicalização é denominado stem. O objetivo desta etapa é garantir uma maior flexibilização na comparação das respostas dos alunos com a do professor, pois palavras distintas, que possuam o mesmo radical, são consideradas pela ferramenta como sendo idênticas.

- Definição de Pesos: Nesta etapa, são definidos os pesos dos stems e este está relacionado à ocorrência do termo, que gerou o stem, no texto.

\subsection{Funcionalidades}

O sistema permite, dentre outras funcionalidades a criação de disciplinas, após o cadastro destas o professor pode acessá-las e então criar testes a elas associados. Quando um novo teste é criado é disponibilizado ao professor a possibilidade adicionar diversos tipos de questões, sendo estas: "questão de múltipla escolha", "questão verdadeira ou falsa", "questão de resposta curta" e "questão discursiva".

Após os alunos responderem às questões, os mesmos possuem feedback imediato sobre seu desempenho, o feedback é provido pela correção automática realizada pela ferramenta. O professor, por sua vez, pode acessar as notas sugeridas pelo sistema para cada questão de forma individual. Entretanto, caso discorde da nota sugerida automaticamente, existe no LMS a possibilidade de que o professor informe sua nota para as questões discursivas, conforme pode ser visualizado na Figura 2.

Desta forma, além de avaliar o aluno, o professor pode analisar sua própria prática pedagógica, pois caso identifique pontos de "fracasso" nos testes respondidos pelos alunos, isso serve como indicador de que pode ser necessária uma revisão dos conteúdos cujas respostas não alcançaram índice satisfatório. 
Figura 2 - Tela de atribuição de notas para questões discursivas.

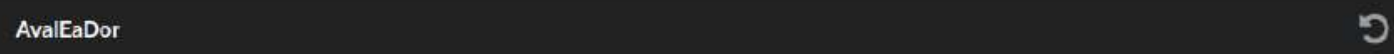

\section{Detalhes das Respostas}

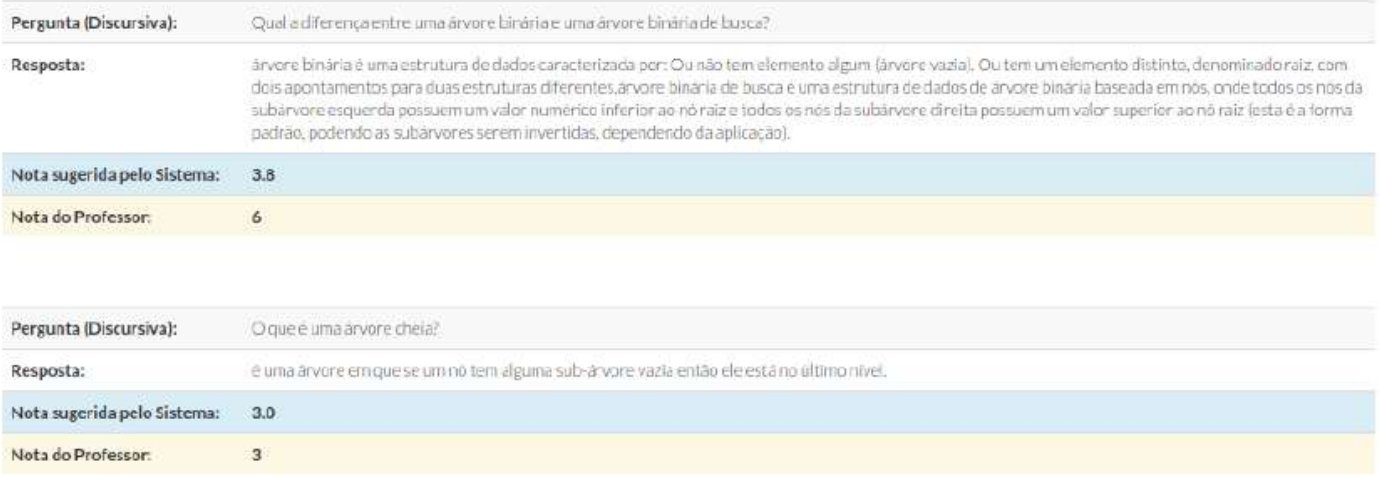

Fonte: Autores.

\subsection{Estudo de Caso}

Para avaliar a pertinência, para o ensino, do LMS desenvolvido optou-se pela realização de um estudo de caso com análise mista, com predominância qualitativa, porém utilizando análises quantitativas em casos específicos. O referido estudo de caso foi realizado a partir da interação de uma amostra composta por noventa e um alunos e onze professores, do Instituto Federal Fluminense campus Bom Jesus do Itabapoana, com o LMS desenvolvido.

Para Yin (2001, p.32) "o estudo de caso é uma investigação empírica de um fenômeno contemporâneo dentro de um contexto da vida real, sendo que os limites entre o fenômeno e o contexto não estão claramente definidos". Ainda segundo o autor o estudo de caso é o método mais utilizado quando há a necessidade de se responder questões do tipo "como" e "por que" e quando o pesquisador possui pouco controle sobre os eventos que ocorrerão no decorrer de sua pesquisa, pois geralmente buscam entender contextos do mundo real.

De forma complementar Pereira et al. (2018) afirmam que os Estudos de Caso são amplamente utilizados em estudos exploratórios, analíticos ou descritivos, sendo que esta metodologia é comumente utilizada quando deseja-se explorar fenômenos individuais ou, processos sociais. Os autores ressaltam ainda que para que um caso possa receber essa denominação, esta deve ter alguma particularidade que o diferencie do habitual, devendo ser analisado da forma mais detalhada e completa possível.

Portanto, o presente estudo caracteriza-se como um estudo de caso, devido à sua característica particular e ao aprofundamento na exploração dos fenômenos gerados por intermédio da utilização da ferramenta, uma vez que além do desenvolvimento da ferramenta em si e utilização de parâmetros conhecidos e quantificáveis para testes desta, foi analisado o comportamento de professores e alunos reais, em situações específicas no uso da ferramenta.

Além disso, optou-se pela utilização do método de análise mista, que combina características dos métodos qualitativo e quantitativo. Segundo Creswell (2007) o método de análise mista é aquele no qual o pesquisado baseia as alegações de conhecimento nos dados coletados de forma qualitativa, para compreender o problema da pesquisa bem como informações numéricas, de forme que a base de dados final represente informações, tanto qualitativa quanto quantitativas. A utilização do método de análise misto pode ser observada ao longo do trabalho, onde são citadas as estratégias para coletas de dados qualitativas, entre os sujeitos da pesquisa e dados quantitativos, obtidos principalmente por intermédio da ferramenta para mensurar o desempenho da ferramenta frente à avaliação realizada por avaliadores humanos.

A interação dos professores consistiu na elaboração de testes, estes compostos por perguntas discursivas e objetivas. 
A interação dos alunos se deu por intermédio da realização dos testes propostos. Após sua conclusão o aluno recebeu feedback acerca do índice de acerto do teste realizado, calculado de forma automática, sem a necessidade de correção do professor. Por fim os professores verificaram se o índice de acerto atribuído automaticamente pelo referido LMS condizia com a correção por ele realizada.

Para coletar as opiniões dos alunos e professores foram criados questionários estruturados com perguntas acerca do LMS utilizado. A estrutura dos questionários foi baseada na escala de verificação de Likert.

Com relação às análises quantitativas, foram utilizados os métodos estatísticos, coeficiente de correlação e teste $t$ com amostras pareadas e uma adaptação do método computacional, validação cruzada, normalmente utilizado em algoritmos de inteligência artificial.

Foi escolhido, para comparação das correções dos professores e sistema, o teste t com amostras pareadas, pois, conforme afirma Fukushima (1998), este tipo de teste é considerado em planejamentos nos quais são realizadas duas medidas de uma mesma unidade amostral, onde a unidade é seu próprio controle. Devendo ser usado quando se deseja mensurar o nível de certa medida antes e depois de uma intervenção.

A adaptação do método validação cruzada foi utilizada com o intuito tornar a avaliação realizada pelo LMS, mais próxima à do avaliador humano. Essa técnica, comumente utilizada em algoritmos de inteligência artificial, consiste na utilização de uma porcentagem dos dados para treinar a ferramenta e o restante dos dados são utilizados para validação. Geralmente os percentuais utilizados são 50\%50\%, 70\%30\% ou 80\%30\% (Akay, 2009, p. 3241).

\section{Resultados e Discussão}

Nesta seção são descritos alguns dos resultados obtidos do estudo de caso realizado para avaliação da pertinência da ferramenta para atividades relacionadas ao ensino. Estes resultados foram obtidos por meio da coleta da opinião dos alunos e professores participantes da pesquisa, realizada por intermédio de questionários, e pela utilização de métodos quantitativos, descritos anteriormente, utilizados na comparação das correções realizadas pelo LMS desenvolvido para esta pesquisa com as realizadas pelos professores.

\subsection{Dados dos questionários}

Para entender quem são os sujeitos da pesquisa e sua percepção acerca de sua interação com a ferramenta desenvolvida para o presente trabalho, optou-se pela utilização de questionários, esta escolha justifica-se pela necessidade de coletar as informações de forma clara e objetiva, porém permitindo aos envolvidos relatarem suas impressões, críticas, elogios e sugestões de melhorias.

Entre outros questionamentos acerca da utilização da ferramenta perguntou-se se "a ferramenta desenvolvida, de maneira geral, contribui para o ensino", se "a utilização da ferramenta pode auxiliar na aprendizagem do aluno", se "os resultados fornecidos pela ferramenta pode indicar pontos onde devo melhorar minha prática pedagógica" e se "de maneira geral a utilização da ferramenta é útil na aplicação de testes/exercícios". Em todos os questionamentos supracitados o retorno foi positivo, indicando que a ferramenta traz contribuições para a prática docente e para atividades de ensino.

Outro aspecto que desejou-se avaliar diz respeito ao melhor uso da ferramenta no cotidiano da prática docente, sendo levantadas inicialmente três possibilidades, "sua utilização em substituição à avaliação tradicional", "sua utilização como complemento à avaliação" e "sua utilização na aplicação de atividades para casa". Neste ponto, a preferência, tanto dos professores quanto dos alunos, foi pela utilização da mesma para atividades para casa, indicando seu potencial em complementação ao conteúdo visto em sala de aula. 


\subsection{Análise do estudo de caso}

Conforme descrito anteriormente, tanto o sistema, quanto o professor da respectiva disciplina, realizaram a correção de todas as questões propostas. Além da comparação realizada pelo professor, foi utilizado o método estatístico, para fins de comparação de médias, "Teste T" com amostras pareadas. Para a realização do "Teste T", considerou-se a utilização padrão, da ferramenta, de $95 \%$ de confiança (ou uma chance de erro de $5 \%$ ).

Inicialmente, das trinta e três questões criadas para o estudo de caso, vinte e duas $(66,6 \%)$ apresentaram diferença significativa entre as médias, ou seja, nos referidos casos, se considera que a correção do sistema e a correção do professor foram estatisticamente distintas, havendo discrepância entre estas. Em onze questões $(33,3 \%)$, a ferramenta apresentou-se, útil em substituição à avaliação realizada pelo professor, ratificando a percepção professores quanto à acurácia da ferramenta, coletadas por intermédio dos questionários.

Entretanto, a diferença entre as médias não é algo exclusivo entre uma ferramenta de Text Mining e a avaliação realizada por um professor. Vianna (2013) afirma que pode haver diferença significativa entre correções de professores distintos para a mesma questão discursiva, pois alguns fatores, alguns irrelevantes ou estranhos ao processo de avaliação, tendem a afetar na decisão dos julgadores.

Apesar de algumas questões terem sido discrepantes, foram observados padrões nas correções que indicaram que estratégias poderiam ser utilizadas para um melhor desempenho da correção automática. Para este fim foi utilizada uma adaptação da técnica de validação cruzada para calibração da ferramenta.

A calibração consistiu na utilização de $30 \%$ das notas das correções em cada questão, porcentagem também utilizada em outros trabalhos como em Reis et al. (2016), notas estas escolhidas aleatoriamente, a fim de produzir uma constante, posteriormente somada à nota sugerida pelo sistema, resultante da média das diferenças entre as notas sugeridas pelos professores e as notas sugeridas pelo sistema, ou seja:

$$
\text { Calibração }=\frac{\Sigma p}{n p}-\frac{\Sigma s}{n s}
$$

Onde "p" representa as notas atribuídas pelo professor responsável pela correção, "s" as notas atribuídas pelo sistema, "np" representa o número total de questões avaliadas pelo avaliador humano e "ns" representa o número total de questões avaliadas pelo sistema. Após a aplicação do método supracitado, o número de questões com diferença significativa reduziu de vinte e duas questões $(66,6 \%)$ para apenas dez questões (30,3\%). Com isso observa-se uma aproximação da média do professor para a média do sistema e uma consequente diminuição das diferenças entre as médias.

\section{Considerações Finais}

Quando considerado o aspecto de usabilidade, conforme foi aferido por intermédio dos questionários aplicados entre os sujeitos da pesquisa, a ferramenta obteve boa aceitação tanto entre os docentes quanto entre os discentes, que a consideraram simples e objetiva nas funcionalidades apresentadas. Além disso, para as atividades de ensino de maneira geral, esta foi considerada bastante pertinente. Especificamente com relação ao desempenho desta em uso em avaliações, em questões do tipo "múltipla escolha", "verdadeiro e falso" e "resposta curta", teve desempenho adequado para os critérios utilizados nestes tipos de questão.

No que tange à correção automática de questões discursivas, esta apresentou resultados satisfatórios quando combinados às avaliações de avaliadores humanos, sendo possível afirmar que a ferramenta possui potencial para este fim, desde que se adotem procedimentos de calibração da mesma. Quando utilizada da forma anteriormente descrita, o desempenho 
desta em aproximadamente dois terços das questões avaliadas não houve diferença significativa entre as avaliações realizadas por humanos e o sistema desenvolvido.

Entretanto, apesar dos resultados obtidos, a construção de uma base de conhecimento com respostas padrão e a participação do professor no processo avaliativo são de extrema importância para obtenção de resultados mais próximos à realidade. Pois como afirma Santos (2016), a maior parte das ferramentas desenvolvidas para correção automática de questões discursivas, depende de um conjunto de respostas já avaliadas para treinamento para obter bons resultados, pois entende-se que desenvolver uma ferramenta integralmente autônoma para correção deste tipo de questão não é simples, pois existem fatores subjetivos que tornam essa uma atividade complexa.

Como proposta de trabalhos futuros, os autores sugerem a possibilidade da combinação da técnica de Text Mining, utilizada no presente trabalho com técnicas de Processamento de Linguagem Natural para obtenção de melhores resultados na contextualização das respostas fornecidas pelos alunos, ou ainda a utilização de técnicas de Inteligência Artificial, como Redes Neurais Artificiais, que permita que a ferramenta seja aprimorada a medida que vai sendo utilizada, e possa até mesmo considerar respostas de alunos, que inicialmente tenham sido avaliadas pela ferramenta de forma insatisfatória, de forma equivocada, como respostas alternativas àquela informada previamente como resposta padrão, fornecendo resultados mais fidedignos à avaliação humana.

\section{Referências}

Akay, M. F. Support vector machines combined with feature selection for breast cancer diagnosis. Science Direct. 3240-3247. https://www.sciencedirect.com/science/article/abs/pii/S0957417408000912.

Alves, A. L., Santos, L. S. \& Oliveira, K. E. J. Educação a distância: feedback interacional por meio do ava. In: Encontro Internacional de Formação de Professores e Fórum Permanente de Inovação Educacional, 9.

Associação Brasileira de Educação a Distância (ABED). Censo EaD.BR: Relatório Analítico da Aprendizagem a Distância no Brasil 2015 = Censo EaD.BR: Analytic Report of Distance Learning in Brazil 2015. Organização ABED - Associação Brasileira de Educação a Distância, Tradução de Maria Thereza Moss de Abreu. Curitiba: InterSaberes, 2016.

Clementino, A. Didática intercomunicativa em cursos online colaborativos. 2008. 268 f. Tese (Doutorado) Curso de Educação, Universidade de São Paulo, São Paulo, 2008. 2008. 268 f. Tese (Doutorado) - Curso de Educação, Universidade de São Paulo.

CGI.br/NIC.br, Centro Regional de Estudos para o Desenvolvimento da Sociedade da Informação (Cetic.br), Pesquisa sobre o Uso das Tecnologias de Informação e Comunicação nos domicílios brasileiros - TIC Domicílios 2018.

Creswell, J. W. Projeto de pesquisa. Métodos qualitativo, quantitativo e misto (2a ed.), tradução de Luciana de Oliveira da Rocha. ARTMED.

Silva, V M. Revisão sistemática da evolução MVC na base ACM. 15² Concurso de Trabajos Estudiantiles, EST 2012.

Dunlosky, J., Rawson, K. A., Marsh, E. J., Nathan, M. J. \& Willingham, D. T. Improving Students' Learning With Effective Learning Techniques. Psychological Science In The Public Interest, 14, 4-58. SAGE Publications. http://dx.doi.org/10.1177/1529100612453266.

Figueira, A. S., Silva, A. G., Melo, B. M., Lino, A. P., Lobato, F. R. L., Morais, A. S. \& Favero, E. L. Module of evaluation automatic essay questions on virtual learning environment LabSQL". In: 8th Iberian Conference on Information Systems and Technologies (CISTI), 2013, p. 510-514, Lisboa, Portugal

Foly, L. S. Uma Arquitetura para Aferir o Conhecimento do Aluno em um Ambiente de Aprendizagem. 2010.73 f. Dissertação (Doutorado) - Curso de Mestrado em Pesquisa Operacional e Inteligência Computacional, Universidade Candido Mendes, Campos dos Goytacazes, 2010

Foly, L. S., Gomes, G. R. R. Uma Arquitetura de Ambiente de Aprendizagem para aferir o conhecimento do aluno. Revista Brasileira de Informática na Educação, 19, 55-62. Sociedade Brasileira de Computação - SB. http://dx.doi.org/10.5753/rbie.2011.19.01.55.

Fukushima, J. T. Estatística Médica. Braz. J. Nephrol, 20, 381-382. https://bjnephrology.org/wp-content/uploads/2019/12/jbn_v20n3a30.pdf

Gomes, G. R. R. Integração de Repositórios de Sistemas de Bibliotecas Digitais e de Sistemas de Aprendizagem. 2006.143 f. Tese (Doutorado) - Curso de Pós-Graduação em Informática, Pontifícia Universidade Católica, Rio de Janeiro, 2006.

Pereira A. S. et al. (2018). Metodologia da pesquisa científica. UFSM. https://repositorio.ufsm.br/bitstream/handle/1/15824/Lic_Computacao_MetodologiaPesquisa-Cientifica.pdf?sequence $=1$

Pinheiro, E. G. (2000). Castells, Manuel. A sociedade em rede. Paz e Terra, 1999. 1. Informação \&Amp, Sociedade: Estudos, 10(2).

Reis, E. R., Castro, P. A. L. \& Sichman, J. S. Enhancing Classification Accuracy Through Feature Selection Methods. In: XIII Encontro Nacional de Inteligência Artificial e Computacional, 2016, Recife. SBC, 2016. 
Research, Society and Development, v. 10, n. 8, e32710817424, 2021

(CC BY 4.0) | ISSN 2525-3409 | DOI: http://dx.doi.org/10.33448/rsd-v10i8.17424

Santos, J. C. A. Avaliação automática de questões discursivas usando LSA. 2016. 117 f. Tese (Doutorado) - Universidade Federal do Pará, Instituto de Tecnologia, Belém, 2016. Programa de Pós-Graduação em Engenharia Elétrica.

Scaico, P. D., Corlett, E. F., Paiva, L. F., Raposo, E. H. S. \& Alencar, Y. Relato da Utilização de uma Metodologia de Trabalho para o Ensino de Ciência da Computação no Ensino Médio. In: Workshop De Informática Na Escola, 18., 2012, Rio de Janeiro. Anais [...]. [S.I.], 2012.

Silva, V. G. \& Gomes, M. J. Usabilidade técnica e usabilidade pedagógica em mobile learning: um estudo de revisão sistemática. In: IV Congresso Internacional Tic Na Educação. 2016, Lisboa. Atas. Ticeduca,. 1817 - 1830.

UNESCO, 2014. O Futuro da aprendizagem móvel: implicações para planejadores e gestores de políticas. Brasília: UNESCO, 2014. 64 p. (Documentos de trabalho da UNESCO sobre aprendizagem móvel).

Vianna, H. M. Flutuações de julgamentos em provas de redação. Cadernos de Pesquisa, 19, 5-9

Yin, Robert K. Estudo de caso - planejamento e métodos. (2a ed.), Bookman. 2001 